\title{
Colonial Competitive Optimization Sliding Mode Controller with Application to Robot Manipulator
}

\author{
Amin Jalali \\ Department of Maritime Electronic and Communication Engineering, College of Maritime Engineering, Chabahar \\ University, Iran \\ E-mail: Max.Jalali@gmail.com
}

Farzin Piltan

Senior Researcher at Research and Development Unit, Sanatkadehe Sabze Pasargad Company, (S.S.P. Co), Shiraz, Iran, WWW.IRANSSP.COM

E-mail: Piltan_f@iranssp.com

\begin{abstract}
Maziyar Keshtgar
Department of Biomedical Engineering, California Polytechnic State University, San Luis Obispo, California E-mail: mkeshga@calpoly.edu
\end{abstract}

\author{
Meysam Jalali \\ Department of Electronic Engineering, University of Sistan and Balouchestan, Iran \\ E-mail: Meysam.Jalali.8812723@gmail.com
}

\begin{abstract}
One of the best nonlinear robust controllers which can be used in uncertain nonlinear systems is sliding mode controller (SMC), but pure SMC results in chattering in a noisy environment. This effect can be eliminated by optimizing the sliding surface slope. This paper investigates a novel methodology in designing a SMC by a new heuristic search, so called "colonial competitive algorithm "in order to tune the sliding surface slope and the switching gain of the discontinuous part in SMC structure. This process decreases the integral of absolute errors which results in tracking the desired inputs by the outputs in designing a controller for robot manipulator. Simulation results prove that the optimized performance obtained through CCA significantly reduces the chattering phenomena and results in better trajectory tracking compared to typical trial and error methods.
\end{abstract}

Index Terms - Uncertain Nonlinear Systems, Evolutionary Algorithm, Classical Control, Sliding Mode Controller, Robot Manipulator, Colonial Competitive Algorithm, Optimization

\section{Introduction and Motivation}

SMC is one of the most powerful nonlinear robust controllers used in a variety of applications. It is composed of variable structure with the switching output in a distinct state space [1]. Stability and robustness are two main characteristics of this controller.
However, the drawback for this controller is the chattering phenomenon caused by a noisy environment. To overcome this problem several works were proposed [2-3].To illustrate, the consideration of boundary layer near the switching surface was proposed [4]. One of the best techniques to overcome this challenge is the use of soft computing (SC) methods in classical controller switch leads to introduction of intelligent agents integrated with SMC algorithm [5-9, 26-31].

Soft Computing (SC) was introduced by Prof. Lotfi Zadeh as one of the intelligent technologies and has been applied to variety of problems widely. SC technologies consist of neural networks (NNs), fuzzy logic (FL), and probabilistic reasoning (PR) which includes belief networks, evolutionary computation (EC), chaos theory, and parts of learning theory. These patterns imitate human intelligence and smart optimization mechanisms detected in the nature [10, 11].The implementation of SC algorith $\mathrm{ms}$ in SMC can provide good trajectory performance which can be conducted by well tuning the controller's parameters [12].

In this paper we apply a colonial competitive algorith m (CCA) [13-14] in designing an optimal SMC. A socio-political competition among the empires serves as the main strategy in CCA for achieving the optimu $m$ result in SMC parameters. Similar to other evolutionary algorith ms that use an initial population, CCA begins with initial empires. Each empire consists of individual countries. There are two different types of countries: colonies and imperialists. CCA constitutes a colonial 
competition among the empires in which the weakest empires collapse. The competitions continue to a point so that there would be only one empire with its colonies while the colonies have the same cost as the imperialist. In designing SMC for robot manipulator, CCA is utilized in optimizing the SMC parameters. The controller achieved from CCA is compared with the typical trial and error method.

Robotic mechanisms are a set of rigid bodies known as links connected by joints. The proposed controller is applied to PUMA-560 which is an academic and industrial 6 DOF serial robot manipulator. Dynamic parameters of PUMA 560 robot manipulator have been identified and written [15].

As this paper follows, section II provides a brief description about the robot model of robot manipulator, studies classical Sliding Mode Controller (SMC) and gives an introduction about Colonial Competitive Algorithm (CCA), section III studies the proposed methodology of tuning SMC with socio-political Imperialist Competitive Algorithm (ICA), section IV demonstrates simulation results, and finally section $\mathrm{V}$ investigates the conclusion.

\section{Theory}

Dynamic Formulation of robot manipulator: The dynamical equation for an n-link robot manipulator is as following $[6,8,9,17,22,23,26-31]$ :

$$
\mathbf{M}(\mathbf{q}) \ddot{q}+\mathbf{C}(\mathbf{q}, \dot{q}) \dot{q}+\mathbf{G}(\mathbf{q})+\tau_{d}=\tau
$$

Where, $M(q) \in \boldsymbol{R} n \times n$ is symmetric and positive inertia matrix, and $q, \dot{q}, \ddot{q} \in \boldsymbol{R} n$ represent the position, velocity, and angular acceleration of the robot joint, respectively. $C(q, \dot{q}) \in \boldsymbol{R} n \times n$ is the matrix of Coriolis and Centrifugal forces.The matrix of $G(q) \in \boldsymbol{R} n \times 1$ is the gravity vector, $\tau_{d} \in \boldsymbol{R} n$ is the bounded disturbance vector and $\tau € \boldsymbol{R} n$ is the control input vector.

Any changes in nonlinear parameters such as Coriolis and Centrifugal functions can cause changes in velocities and subsequently in position. In this case, the robot dynamic equation known as configuration space can be demonstrated as:

$$
\tau=M(q) \ddot{q}+B(q) \cdot[\dot{q} \dot{q}]+C(q) \cdot\left[\dot{q}^{2}\right]+G(q)
$$

Where, B (q) $€ \mathbf{R}^{\mathrm{n} \times \mathrm{n}(\mathrm{n}-1) / 2}$ indicates matrix of Corio lis torque and $C$ (q) $\in \mathbf{R}^{\mathrm{n} \times \mathrm{n}}$ displays matrix of Centrifugal torques.

Some researches perform either the summation of Lagrange's formulation or the Gibbs-Alembert formulation to acquire the kinetic energy matrix and gravity vector which can be used to derive the dynamic modeling of the robot manipulators [2, 3, 8-16]:

$$
\begin{aligned}
& \ddot{\boldsymbol{q}}=\boldsymbol{M}^{-1}(\boldsymbol{q}) .\{\boldsymbol{\tau}-[\boldsymbol{B}(\boldsymbol{q}) .[\dot{\boldsymbol{q}} \dot{\boldsymbol{q}}]+ \\
& \left.\left.\boldsymbol{C}(\boldsymbol{q}) .\left[\dot{\boldsymbol{q}}^{2}\right]+\boldsymbol{G}(\boldsymbol{q})\right]\right\}
\end{aligned}
$$

This technique is very effective since the nonlinear and coupled robot manipulator dynamics are replaced by a linear and decoupled second order system. In this paperfirst 3 degrees of freedom PUMA 560 robot manipulator is modeled and discussed.

Classical sliding mode controller for robot manipulator: sliding mode controller description, problem statement and some solutions was presented in literature [15-21, 26-31].We can write the control law of SMC for six degrees of freedom PUMA-560 classical robot manipulator as [8]:

$$
\tau=\tau_{\text {sat }}+\tau_{\text {equ }}
$$

The discontinuous part of total torque so called saturation component ( $\left.\tau_{\text {sat }}\right)$ and computed as:

$$
\tau_{\text {sat }}=k . s \operatorname{at}(\mathbf{s} / \varphi)
$$

Where $S$ is the time varying sliding surface, $\boldsymbol{\varphi}$ is the boundary layer thickness, sat () is the saturation function and the $\mathbf{k}$ is the switching gain which is positive and determined by:

$$
\begin{aligned}
& \mathbf{S}=\dot{\mathbf{e}}+\lambda * \mathbf{e}+(\lambda / 2)^{2} \int \mathbf{e ~ d t} \\
& \left(\mathbf{e}=\mathbf{q}_{\text {desired }}-\mathbf{q}_{\text {actual }}\right)
\end{aligned}
$$

erepresents error rate, $\boldsymbol{\lambda}$ is a coefficient and $\operatorname{sat}(\mathrm{s} / \varphi)$ can be written as:

$$
\left\{\text { sat }\left(\frac{s}{\emptyset}\right)\right\}_{i}= \begin{cases}\frac{s_{i}}{\left|s_{i}\right|} \quad \text { if }\left|s_{i}\right| \geq \varphi_{i} \\ \frac{s_{i}}{\emptyset_{i}} & \text { if }\left|s_{i}\right| \leq \varphi_{i}\end{cases}
$$

And the model-based component $\left(\tau_{\text {equ }}\right)$ represents the nominal dynamics of systems which can be calculated as follows:

$$
\tau_{\text {equ }}=\left[\mathbf{M}^{-1}(\mathbf{B}+\mathbf{C}+\mathbf{G})+\dot{S}\right]^{*} \mathbf{M}
$$

Where, $\dot{\boldsymbol{S}}$ is the derivation of the sliding surface and will be written as:

$$
\begin{aligned}
& \dot{\mathbf{S}}=\ddot{\boldsymbol{\theta}}+\boldsymbol{\lambda} * \dot{\mathbf{e}}+\left(\frac{\lambda}{2}\right) * \mathbf{e} \\
& \left(\boldsymbol{\theta}=\mathbf{q}_{\text {desired }}\right)
\end{aligned}
$$

The expression for control output by replacing (5) and (8) in (4) can be written as:

$$
\begin{aligned}
& \tau=\left[\mathbf{M}^{-1}(\mathbf{B}+\mathbf{C}+\mathbf{G})+\dot{\mathbf{S}}\right] * \mathbf{M}+ \\
& \text { k. } \operatorname{sat}(\mathbf{s} / \varphi)
\end{aligned}
$$


Colonial Competitive Algorithm (CCA): Imperialism is the standpoint of developing power and control by a government to further increase its own territory. A country may rule others out by either direct control or less obvious means such as a control of markets for goods or raw materials which is so called neocolonialis m [24]. Colonial Competitive Algorithm (CCA) is a new global heuristic and initiative search that utilizes imperialism and imperialistic contest process as an origin of inspiration. E. Atashpaz- Gargari and C. Lucas presented the hierarchical steps of CCA as following [13, 14]:

1) Select some random points on the function and initialize the empires.

2) Move the colonies toward their relevant imperialist (Assimilating).

3) If there is a colony in an empire which has lower cost than that of imperialist, exchange the positions of that colony and the imperialist.

4) Compute the total cost of all empires (Related to the power of both imperialist and its colonies).

5) Pick the weakest colony (colonies) from the weakest empire and give it (them) to the empire that has the most likelihood to possess it (Imperialistic competition).

6) Eliminate the powerless empires.

7) If there is just one empire, stop, if not go to 2.

The procedure of process begins with an initial population. Any individual of the population is called a country. Some of the best countries in the population are chosen to be the imperialist states, and all the other countries constitute the colonies of these imperialists. All the colonies of initial population are apportioned among the imperialists on the basis of their power which are contrarily in harmony with their cost and begin moving toward their related imperialist country. This act of moving is an easy pattern of assimilation action that was investigated by some imperialist states [25] which depicts in Figure 1.

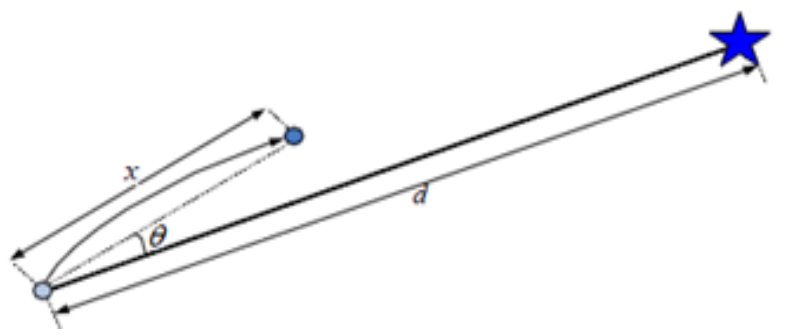

Fig. 1: Motion of Colonies toward their relevant imperialist

In this movement, $\theta$ and $\mathrm{x}$ are rando $\mathrm{m}$ numbers with uniform distribution, and $\mathrm{d}$ is the distance between the colony and the imperialist. $\mathrm{X} \sim \mathrm{U}(0, \beta \times \mathrm{d}) \theta \sim \mathrm{U}(-\gamma, \gamma)$ $\beta$ and $\gamma$ are arbitrary numbers that adjust the area that colonies randomly search around the imperialist. In our implementation $\beta$ and $\gamma$ are 2 and $\pi / 4(\mathrm{Rad})$ respectively.

The total power of an empire is the aggregate of power of the imperialist country and the power of its colonies. Any empire that is not able to prosper in imperialist competition and cannot enhance its power or at least prevent diminishing its power will be removed. The imperialistic competition will progressively yield a growth in the power of great empires and a reduction in the power of weaker ones. Feeble empires will misplace their power gradually and eventually they will collapse.

This act of moving of colonies toward their related imperialists along with competition among empires and also breakdown and collapse mechanism will expectantly bring about all the countries to converge to one empire in the world and all the other countries will be its colonies. In this perfect new world colonies have the same position and similar power as the imperialist.

\section{Methodol ogy}

The output of an ideal SMC has some defined characteristics such as having a precise tracking trajectory, stability, robustness, resistivity against the perturbations, least changes to the dynamic parameters, and lack of any chattering phenomenon in the torque. The standard approach to SMC is based on formulation (10).

Luanda $(\lambda)$ in formulations (6), (9) and $(\mathrm{K})$ in formulation (10) are coefficients which have the prominent impact on discontinuous portion $\left(\tau_{\text {sat }}\right)$. The saturation function (sat(S)) performs the switching procedure. External disturbances influence on the tracking trajectories, error rate, and torque which result in chattering phenomenon. These high oscillations with a small range of motion exist in all systems. In order to achieve a desirable performance by SMC, it is required to tune the two parameters $(\lambda)$ and $(K)$.

In this paper, the applied methodology chooses the best values for these deterministic coefficients by utilizing the colonial competitive algorithm (CCA). This algorithm optimizes the parameters $(\lambda$ and K) in order to determine the appropriate values for them. In ICA algorith $\mathrm{m}$, the integral absolute error is used in cost function to calculate the minimum cost function for each decade. The ICA algorith $m$ is applied to SMC by thirty initial countries and five initial imperialist countries.

The competition process can be reviewed in the Figure.2, where the mean and minimum costs of all imperialists versus iteration are depicted. Parameters of SMC obtained by CCA and typical trial and error methods are demonstrated in Table 1. 
Table 1: The value of parameters for ICA-SMC and SMC

\begin{tabular}{|c|c|c|}
\hline Parameters & ICA-SMC & Typical SMC \\
\hline Luanda $\left(1^{\text {st }}\right.$ link $)$ & 8.0897 & 5 \\
\hline Luanda $\left(2^{\text {nd }} l i n k\right)$ & 5.5722 & 7 \\
\hline Luanda $\left(3^{\text {rd }} l i n k\right)$ & 9.0268 & 5 \\
\hline $\mathrm{K}\left(1^{\text {st }}\right.$ link $)$ & 43.0758 & 14 \\
\hline $\mathrm{K}\left(2^{\text {nd }}\right.$ link $)$ & 63.1662 & 27 \\
\hline $\mathrm{K}\left(3^{\text {rd }}\right.$ link $)$ & 38.3783 & 13 \\
\hline
\end{tabular}

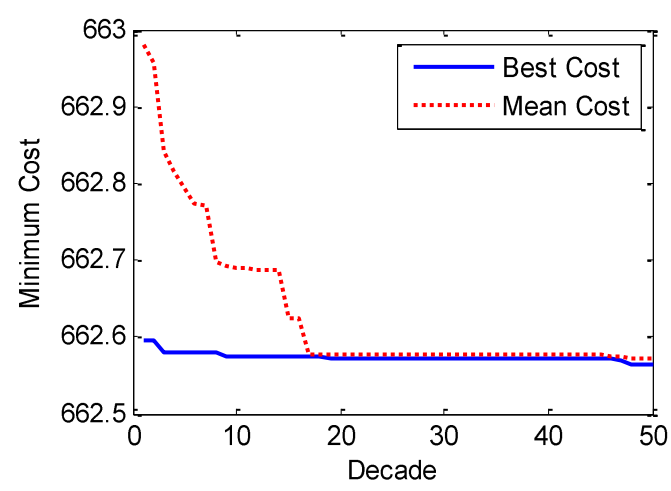

\section{Simulation Results:}

Optimal sliding mode controller using colonial competitive Algorithm (ICA-SMC) and typical trial and error sliding mode controller (SMC) were examined for step response trajectories. In this simulation the first, second, and third joints move from initial point to destination position considering with and without external disturbances. The simulations were performed in MATLAB/SIMULINK. The comparisons were accomplished on indices namely, tracking trajectory performance and chattering rejection or robustness (Disturbance rejection).

Tracking Performances: As it is obvious in Figure 3, proposed ICA-SMC and typical SMC have similar tracking performance but ICA-SMC has better settling time in every three links while these is no external disturbances. In second step of examination an unknown output disturbance is applied to system. The results in Figure. 4 display that the proposed ICA-SMC is able to reject the external disturbances and its tracking is stable. The noise amplitude is equal to 50\% of input amplitude and it was exerted simultaneously at the beginning of the process.

Fig. 2: Mean and minimum cost of all imperialists versus iteration

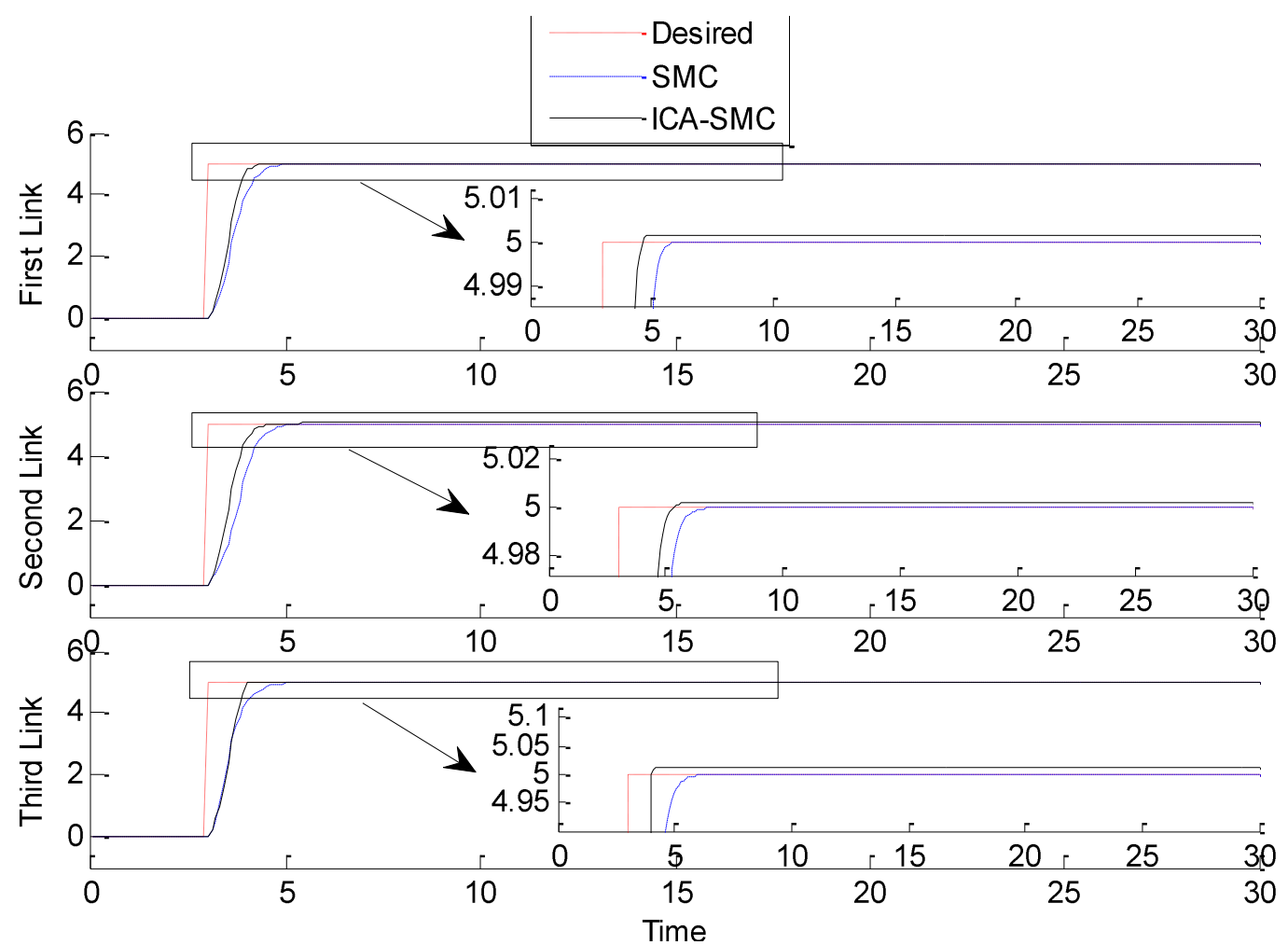

Fig. 3: Step Response of ICA-SMC and SMC for first, second and third link trajectory without external disturbance 


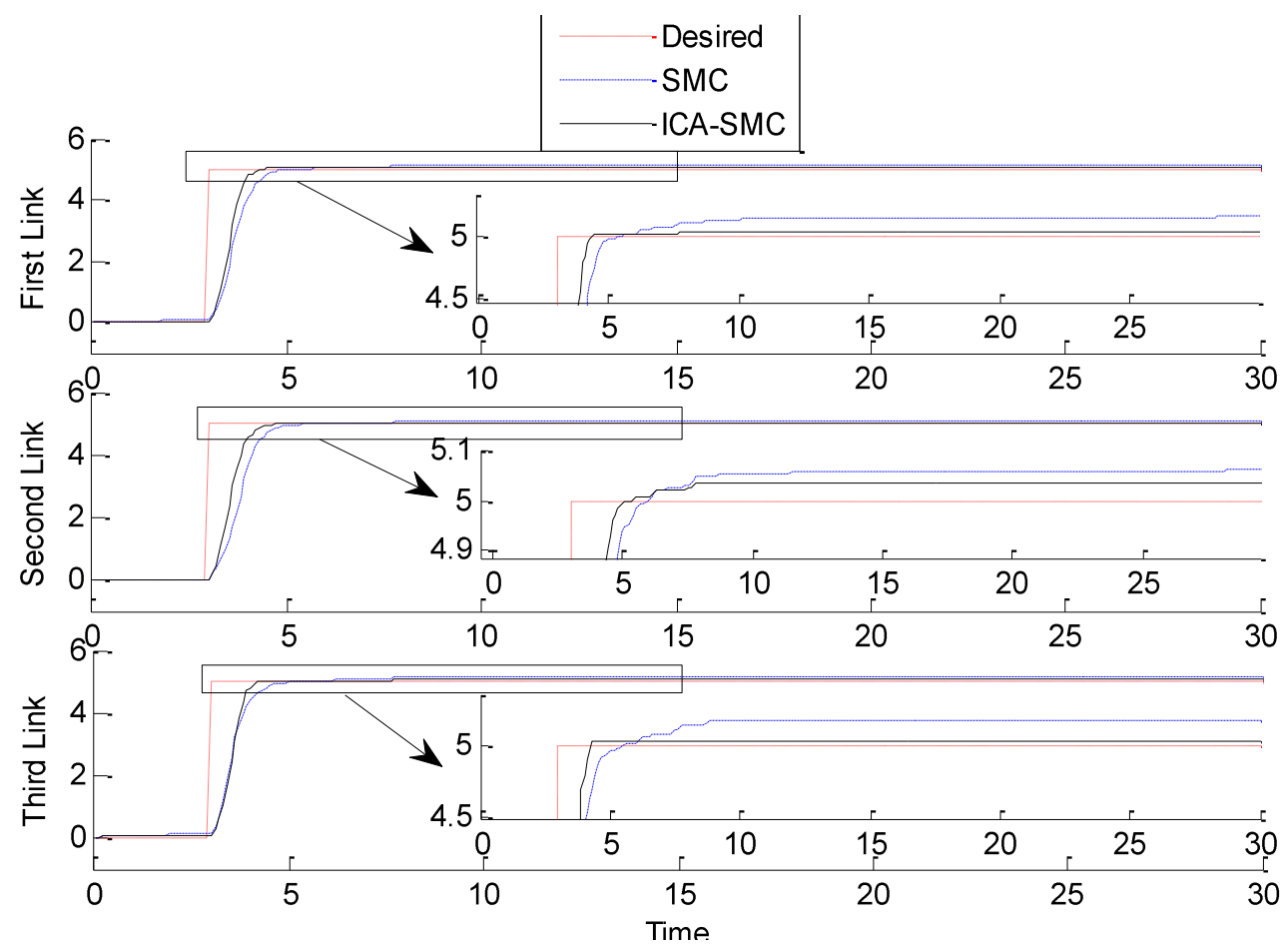

Fig. 4: Step Response of ICA-SMC and SMC for first, second and third link trajectory with external disturbance

Chattering rejection and robustness: Both $\mathrm{SMC}$ and ICA-SMC have similar torque trend when there is no noise (Figure.5).As it was declared, a white noise as an external noise was exerted to system to study the disturbance rejection of it. The results are shown in Figure.6. SMC has oscillation tracking at the presence of disturbances which causes instability and chattering phenomenon while the proposed ICA-SMC displays smoother trend and better performance because it has no chattering phenomenon in its torque and has a stable procedure.
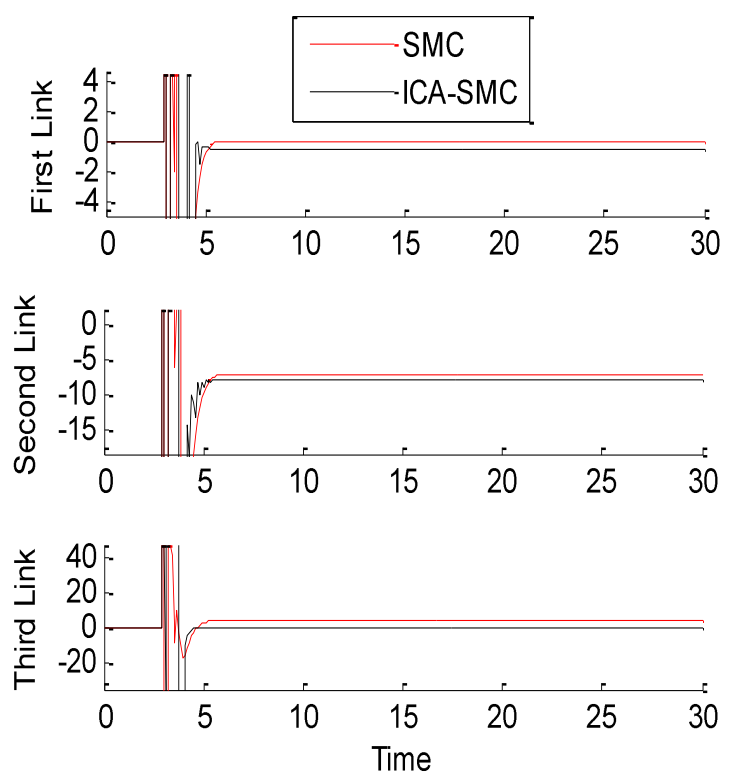

Fig. 5: Torque Profiles without External Disturbances for all links
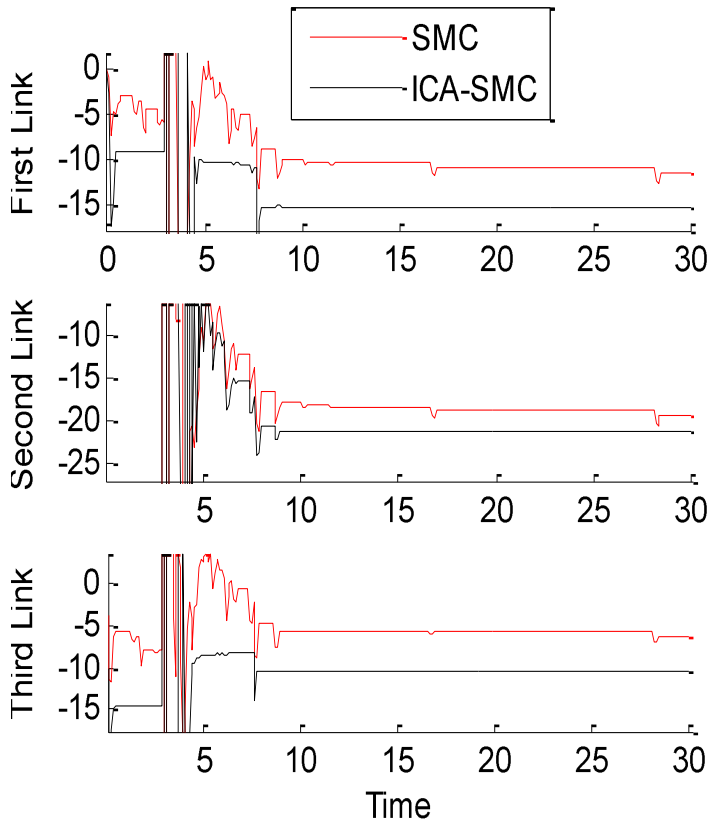

Fig. 6: Torque trend and chattering rejection test to investigate the robustness for all links

\section{Conclusion:}

This paper provides a novel approach in designing an optimal SMC by Imperialist Competitive algorithm applied to PUMA 560 industrial robot manipulator. This new heuristic search tunes the sliding surface slope and the switching gain of the saturation torque in SMC framework for the best output determined in cost function. The cost function calculates all possible responses with the min imumerror rate and then chooses 
the best answer. Simulation results verify that the suggested socio political ICA-SMC considerably lessens the chattering phenomena rather than typical SMC because ICA has optimized and well-tuned SMC's parameters to obtain the best performance. Subsequently the best possible tracking trajectory is acquired. The proposed ICA-SMC has robustness and stability to external disturbances. Moreover it has smoother torque and trajectory without any high oscillation movements (chattering).

\section{References:}

[1] Utkin, I.V., 1977. Variable structure systems with sliding modes, IEEE. Trans. Auto. Control, 22(2): 2121-222.

[2] H. Elmali and N. Olga, Robust output tracking control of nonlinear MIMO systems via sliding mode technique, Automatica 28 (1992) 145-151.

[3] C.L. Hwang and C.H.Lan, the position control of electrohydraulic servomechanism via a novel variable structure control, Mechatronic 4 (1994) 369-391.

[4] J.-J.E. Slotine, Sliding controller design for nonlinear systems, International Journal of Control 40 (2) (1984) 421-434.

[5] T. R. Kurfess, Robotics and automation handbook: CRC, 2005.

[6] B. Siciliano and O. Khatib, Springer handbook of robotics: Springer-Verlag New York Inc, 2008.

[7] OKYAY KAYNAK., 2001. Guest Editorial Special Section on Computationally Intelligent Methodologies and Sliding-Mode Control. IEEE TRANSACTIONS ON INDUSTRIAL ELECTRONICS, 48(1): 2-3.

[8] Piltan, F., et al.,Design Sliding Mode Controller for RobotManipulator withArtificialTunable Gain, Canadian Pure and Applied Sciences, Vol.5,No.2,pp.1573-1579,June 2011.

[9] Piltan, F., et al.,Artificial ControlofNonlinearSecondOrderSystems

BasedonAFGSMC, AustralianJournalof Basic andApplied Sciences, 5(6):509-522,2011.

[10] The Berkeley Institute in Soft Computing. [Online] Available:http://www-bisc.cs.berkeley.edu

[11] Xinghuo Yu, Fellow, IEEE, and O. Kaynak, Fellow, IEEE, Sliding-Mode Control with Soft Computing: A Survey, IEEE TRANSACTIONS ON INDUSTRIAL ELECTRONICS, VOL. 56, NO. 9, SEPTEMBER 2009.

[12] A. Sabanovic, K, Jezernik and O. Kaynak, Chattering free sliding modes in robotic manipulators control, Mechatronics 1 (1994).
[13] E.Atashpaz-Gargari, C. Lucas, Imperialist Competitive Algorithm: An Algorithm for Optimization Inspires by Imperialistic Competition, 2007 IEEE Congress on Evolutionary Computation, Sigapore, 2007.

[14] E.tashpaz-Gargari, C. Lucas, "Colonial Competitive Algorithm: A Socio-Politically Inspired Optimization Strategy,IEEE transaction on Evolutionary Computation.

[15] Brian Armstrong, OussamaKhatib\& Joel Burdick, "The Explicit Dynamic Model and Inertial Parameters of the PUMA 560Arm" , Stanford University, Artificial Intelligence Laboratory, IEEE 1986.

[16] Slotine, J.J.E. and J.A. Coetsee, 1986. Adaptive sliding controller synthesis for nonlinear systems, Int. J. Control, 43(6): 1631-1651.

[17] Slotine, J.J.E. and W. Li, 1987. On the adaptive control of robot manipulators, Int. J. Robotics research, 6(3): 49-59.

[18] Armstrong, B., Khatib, O. and Burdick, J. 2002. The Explicit Dynamic Model and Inertial Parameters of the PUMA 560 Arm. IEEE, 510-518.

[19] ZHANG D. Q. and Panda S. K. Chattering-free and fast response sliding mode controller. IEE Proceedings-D:Theory and Applications, 1999, 146: 171-177.

[20] Wang, L.X., 1993. Stable adaptive fuzzy control of nonlinear systems. IEEE Trans. Fuzzy systems, 1(2): 146-154.

[21] SPONG M W, VIDIASAGAR M. Robot Dynamics and Control, Wiley, New York, 1989.

[22] Abdel-Razzak MERHEB, "Nonlinear Control Algorithms applied to 3 DOF PUMA Robot", METU 2008.

[23] The Hutchinson Dictionary of World History, Oxford: Helicon Publishing, 1999.

[24] R. R. Palmer, "A History of the Modern World", New York: Alfred A. Knopf, 1964, (C)1956.

[25] Piltan, F., et al.,Design of Model Free Adaptive Fuzzy Computed Torque Controller: Applied to Nonlinear Second Order System,International Journal of Robotics and Automation (IJRA), Volume (2): Issue (4): 2011.

[26] Piltan, F., et al.,Design of PC-Based Sliding Mode Controller and Normalized Sliding Surface Slope Using PSO Method for Robot Manipulator, International Journal of Robotics and Automation (IJRA), Volume (2): Issue (4): 2011.

[27] Piltan, F., et al., Novel Artificial Control of Nonlinear Uncertain System: Design a Novel Modified PSO SISO Lyapunov Based Fuzzy Sliding Mode Algorithm, International Journal of 
Robotics and Automation (IJRA): Volume (2): Is sue (5): 2011.

[28] Piltan, F., et al.,Evolutionary Design of Mathematical Tunable FPGA Based MIMO Fuzzy Estimator Sliding Mode Based LyapunovAlgorithm: Applied to Robot Manipulator," International Journal of Robotics and Automation (IJRA), Volume (2): Is sue (5): 2011.

[29] Piltan, F., et al.,Artificial Robust Control of Robot Arm: Design a Novel SISO Back-stepping Adaptive Lyapunov Based Variable Structure Control,International Journal of Control and Automation (IJCA): Vol. 4 No. 4, December, 2011.

[30] Piltan, F., et al., 2011. Design Artificial Nonlinear Robust Controller Based on CTLC and FSMC with Tunable Gain, International Journal of Robotic and Automation, 2 (3): 205-220.

[31] Piltan, F., et al., 2011. Design Mathematical Tunable Gain PID-Like Sliding Mode Fuzzy Controller with MinimumRule Base, International Journal of Robotic and Automation, 2 (3): 146-156.

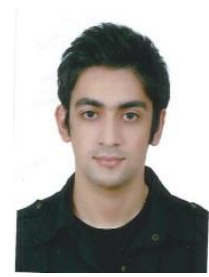

Maziyar Keshtgar is a Biomedical Engineering engineer researcher. His research activities deal with the artificial intelligence control, bioinformatics, orthopedic biomechanics, bone-mechanics, neural networks, and electronics

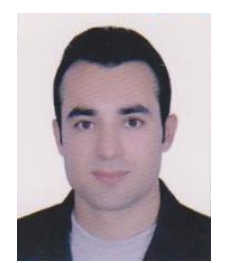

Meysam Jalali is an electronic engineer researcher. His research activities deal with the solid state electronic devices, control, artificial intelligence and expert system.

How to cite this paper: Amin Jalali, Farzin Piltan, Maziyar Keshtgar, Meysam Jalali,"Colonial Competitive Optimization Sliding Mode Controller with Application to Robot Manipulator", International Journal of Intelligent Systems and Applications(IJISA), vol.5, no.7, pp.50-56, 2013. DOI: 10.5815/ijisa.2013.07.07

\section{Authors' Profiles}

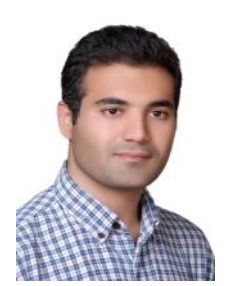

Amin Jalali is an Electrica1/Electronic researcher of research and development company SSP. Co. In 2010 he is jointed the research and development company, SSP Co, Shiraz, Iran and he is also a manager of Rohan Abzar Javdan Mehr corporation. He is the main author of more than 6 scientific papers in refereed journals. His main areas of research interests are nonlinear control and auto mation, artificial intelligence control, optimization, fuzzy theory, neural network, energy systems, and computer vision.

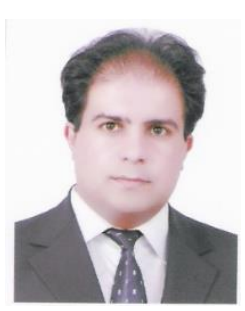

Farzin Piltan was born on 1975, Shiraz, Iran. In 2004 he is jointed the research and development company, SSP Co, Shiraz, Iran. In addition to 7 textbooks, Farzin Piltan is the main author of more than 70 scientific papers in refereed journals. $\mathrm{He}$ is editorial board of international journal of control and automation (IJCA), editorial board of International Journal of Intelligent System and Applications (IJISA), editorial board of IAES international journal of robotics and automation, editorial board of International Journal of Reconfigurable and Embedded Systems and reviewer of (CSC) international journal of robotics and automation. His main areas of research interests are nonlinear control, artificial control system and applied to FPGA, robotics and artificial nonlinear control and IC engine modelling and control. 